\title{
Role of eslicarbazepine in the treatment of epilepsy in adult patients with partial-onset seizures
}

This article was published in the following Dove Press journal:

Therapeutics and Clinical Risk Management

II March 2010

Number of times this article has been viewed

\author{
Martin E Brown \\ Rif S El-Mallakh \\ 'Department of Neurology, \\ ${ }^{2}$ Department of Psychiatry and \\ Behavioral Sciences, University \\ of Louisville School of Medicine, \\ Louisville, Kentucky, USA
}

Correspondence: Rif S El-Mallakh Professor and Director, Mood Disorders Research Program, Department of Psychiatry and Behavioral Sciences, University of Louisville School of Medicine, MedCenter One, 50 I East Broadway, Suite 340, Louisville, Kentucky 40202, USA

$\mathrm{Tel}+\mathrm{I}(502) 852-\mathrm{I}$ I 24

$\mathrm{Fax}+\mathrm{I}(502) 852-5098$

Email rselma0I@louisville.edu

\begin{abstract}
Eslicarbazepine is a new dibenzazepine antiepileptic agent. It is a high affinity antagonist of the voltage-gated sodium channel. It is closely related to both carbamazepine and oxcarbazepine. Eslicarbazepine has similar affinity to inactivated sodium channels (channels in just activated neurons) as carbamazepine, and greater efficacy in animal models of seizure than oxcarbazepine. In human placebo-controlled trials of a single daily dose of eslicarbazepine added to other anti-epileptic agents, significant seizure reductions occurred with 800 and $1200 \mathrm{mg}$ daily, with nearly half of the patients experiencing a greater than $50 \%$ reduction in seizure frequency. Adverse events (AEs) occurred in over $50 \%$ of patients receiving therapeutic doses of eslicarbazepine (compared to $31.4 \%-44.7 \%$ of placebo-treated subjects), but were generally mild or moderate. Eight to $19.6 \%$ of eslicarbazepine treated patients discontinued due to AEs (compared to $3.9 \%-8.5 \%$ of placebo-treated subjects). In these patients receiving combination anticonvulsant therapy, the most common AEs were dizziness, nausea and vomiting, somnolence, and diplopia. Eslicarbazepine is an effective and reasonably well-tolerated adjunct in patients with suboptimal control of their partial seizures.
\end{abstract}

Keywords: eslicarbazepine, licarbazepine, voltage-gated sodium channel, partial-onset seizures, epilepsy

Seizures are among the most common chronic neurologic problems encountered in medical practice. Focal- or partial-onset epilepsy, defined as recurrent, unprovoked episodes of abnormal electrical activity that begin in one part of the brain, is the most common form of epilepsy diagnosed in adults with an annual incidence of 10 to 15 per 100,000 in young- to middle-aged adults and an annual incidence as high as 70 per 100,000 in adults over age $60 .{ }^{1}$ The prevalence of partial onset epilepsy in the United States and Europe has been estimated between 3 and 10 per $1000 .^{2,3}$ The goal of seizure management is complete seizure control with minimal side effects. Quality of life (QoL) studies have suggested that patients who suffer even a single seizure per year have a reduced QoL. ${ }^{4}$ Seizures, especially convulsive seizures, are associated with significant morbidity and mortality, including a higher incidence of accidental drowning, fractures, head and soft-tissue injuries, and serious motor vehicle accidents. ${ }^{5}$ Severe burn injuries are also more likely as patients with epilepsy are overrepresented in burn units. ${ }^{6}$ The economic and social impact of uncontrolled seizures is also significant. Lost productivity due to seizures is a major 'indirect cost' of epilepsy. ${ }^{7}$ Driving restrictions in the United States vary by state with some states prohibiting driving for as long as 12 months after a single seizure. ${ }^{8}$ 
Complete seizure control is not easily achieved. In one large observational study, only $47 \%$ of patients became seizure free following initial monotherapy with an appropriately chosen anti-epileptic drug (AED), and only $63 \%$ of patients ultimately became seizure-free with medical management. ${ }^{9}$ Side-effects of AEDs are common, and frequently contribute to non-compliance. Newer AEDs, while not necessarily more effective at controlling seizures, clearly have fewer drugdrug interactions and may be better tolerated. ${ }^{10}$ Laboratory monitoring for hematologic and metabolic effects is often unnecessary with newer AEDs, although idiosyncratic reactions still occur. On the other hand, potential teratogenicity and effects of long-term therapy are better understood in older AEDs. Given the prevalence of epilepsy which is refractory to currently available medications, new agents with favorable efficacy and tolerability are desirable.

Eslicarbazepine acetate $\left(\right.$ Stedesa $^{\mathrm{TM}}$ in the United States; Zebinix ${ }^{\circledR}$, Exalief $^{\circledR}$ in Europe; chemical name $(S)$-(-)-10-acetoxy-10,11-dihydro-5H-dibenz[b,f]azepine5-carboxamide; development name BIA 2-093), is a new dibenzazepine that has documented efficacy as an adjunct agent in patients with inadequately controlled partial-onset seizures. It is currently being reviewed by the United States Food and Drug Administration and has been approved by the European Medicines Agency (EMEA) in the European Union. Additionally, it is undergoing investigation for monotherapy treatment of partial epilepsy and bipolar illness.

\section{Pharmacology}

Eslicarbazepine is administered as the acetate salt which is rapidly and nearly completely reduced by esterases in the liver to the S enantiomer, S-licarbazepine or eslicarbazepine (BIA 2-005)
(Figure 1). This is the active form in the brain and accounts for approximately $95 \%$ of the metabolic product of the parent prodrug. ${ }^{11}$ The inactive R enantiomer and oxcarbazepine account for less than $5 \%$ of the metabolic product. ${ }^{11,12}$ It is important to note that eslicarbazepine is the active metabolite of oxcarbazepine. Oxcarbazepine is actually a pro-drug that is metabolized into both the $S$ and $R$ enantiomers of licarbazepine at a ratio of 4 or 5 to $1 .^{12,13}$ The nearly complete conversion of eslicarbazepine acetate to the active eslicarbazepine is believed to be the mechanism leading to fewer adverse neurological consequences in rats than oxcarbazepine, ${ }^{14}$ and more consistent anti-seizure effect than oxcarbazepine in a rat electroshock seizure model. ${ }^{12}$

\section{Mechanism of action}

Eslicarbazepine binds avidly and blocks the inactivated voltage-gated sodium channel (VGSC). The VGSC is the major source of sodium entry when a neuron depolarizes, and consequently allows for the action potential to propagate. VGSCs have 3 states. In the deactivated or resting state, the VGSC is closed, but the channel is responsive to a depolarization signal. When the cell depolarizes, the VGSC opens to allow sodium ion entry. After the action potential, the VGSC enters an inactivated state, in which it is closed and not responsive to voltage changes. ${ }^{15}$ Eslicarbazepine binds to the inactivated form of the VGSC and prevents its reversion to the receptive resting or deactivated form. ${ }^{16,17}$ This means that eslicarbazepine binds to more active neurons preferentially. This mechanism is shared by carbamazepine, oxcarbazepine, and other anticonvulsants. ${ }^{1718}$ The affinity of eslicarbazepine to the inactivated form of the VGSC is similar to that of carbamazepine, but its affinity to the resting form of VGSC is some 3 times less. ${ }^{12,17}$ This suggests that eslicarbazepine is less

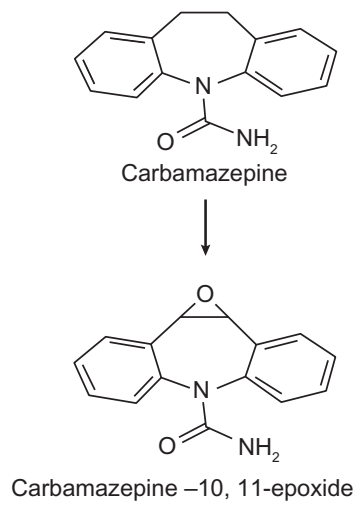

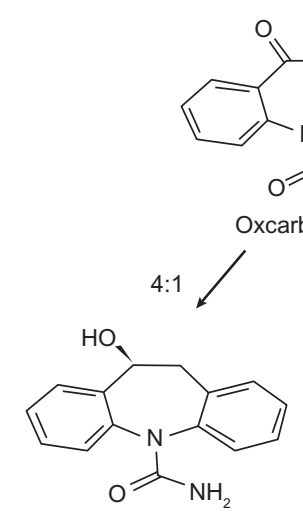

S (+)-licarbazepine

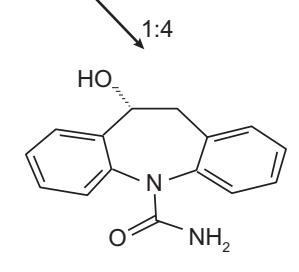

$R(-)$-licarbazepine

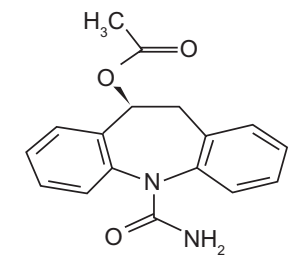

Eslicarbazepine acetate

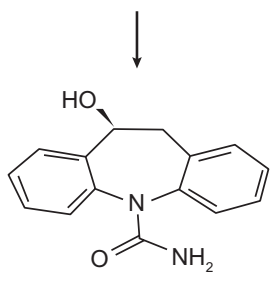

$\mathrm{S}(+)$-licarbazepine

Figure I Eslicarbazepine is formed by reduction of the acetate salt (shown on the right) or may be found as the predominant (4:I) active metabolite of oxcarbazepine (middle). Carbamazepine and its active epoxide metabolite are shown on the left. Reprinted with permission from Almeida L, Soares-da-Silva P. Eslicarbazepine acetate (BIA 2-093). Neurotherapeutics. 2007;4:88-96. ${ }^{12}$ Copyright @ 2007 Elsevier. 
likely to bind to normally active neurons, and therefore, less likely to cause adverse neurological consequences. Indeed when compared with either carbamazepine or oxcarbazepine, eslicarbazepine had less neurologic impairment in rats, ${ }^{14}$ and was less toxic to cultured hippocampal neurons. ${ }^{19}$

\section{Pharmacokinetics}

Bioavailability of eslicarbazepine in a liquid suspension or tablet formulations is equivalent. ${ }^{20}$ Food does not affect the absorption or subsequent drug levels. ${ }^{21,22}$ The possible effect of gender on pharmacokinetics was studied in 24 healthy subjects (12 male) who received eslicarbazepine for 8 days. There were no significant differences in the bioavailability of the drugs, although women absorbed more drug than men. ${ }^{23}$ Finally, age did not affect absorption when young (age $18-40$ years, $\mathrm{n}=12$ ) and older (age $>65$ years, $\mathrm{n}=12$ ) subjects received $600 \mathrm{mg}$ in a single dose. ${ }^{11}$

The time to maximal serum concentration for the therapeutic doses of 900 or $1200 \mathrm{mg} /$ day, or up to $2400 \mathrm{mg} /$ day, ranges from 2.3 to 4 hours. ${ }^{12}$ Both the rate and the area under the curve (AUC) increased in a linear fashion with increasing dose (400-2400 mg/day). The effective half-life of eslicarbazepine is approximately 20 to 24 hours, with steady state being reached in 4 to 5 days. ${ }^{12}$

Eslicarbazepine acetate is metabolized to eslicarbazepine very rapidly in the liver. Levels of the parent drug, eslicarbazepine acetate, are too low to be measureable after single or multiple doses. ${ }^{12}$ Eslicarbazepine is eliminated exclusively by the kidney and $91 \%$ of the drug is recovered in the urine. ${ }^{24}$ Clearance from the plasma occurs at a consistent rate of 20 to $30 \mathrm{~mL} / \mathrm{min} .{ }^{12}$ Approximately $20 \%$ of the dose is recovered in the urine after 12 hours, and $40 \%$ within 24 hours. ${ }^{12,25}$ Subjects with moderate hepatic impairment $(n=8)$ break down eslicarbazepine acetate more slowly, so that its levels can actually be detected, but this difference does not significantly alter the pharmacokinetics. ${ }^{24}$ Subjects with mild renal impairment $(n=8)$ eliminate eslicarbazepine more slowly than healthy controls $(10.2 \mathrm{~mL} / \mathrm{min}$ vs $17.3 \mathrm{~mL} / \mathrm{min}$ in controls), but this not clinically significant ${ }^{26}$ However, subjects with moderate $(\mathrm{n}=8)$ or severe renal impairment $(\mathrm{n}=8)$ clear eslicarbazepine much more slowly $(3.7 \mathrm{~mL} / \mathrm{min}$ and $1.5 \mathrm{~mL} / \mathrm{min}$, respectively). ${ }^{26}$ Eslicarbazepine is cleared with dialysis in subjects with end-stage kidney disease requiring dialysis. ${ }^{26}$

Enantiomer-specific levels can be measured with high performance liquid chromatography separation and ultraviolet light detection method. ${ }^{27}$ Levels at therapeutic doses ranged from $4.7 \pm 4.0 \mu \mathrm{g} / \mathrm{mL}$ for $800 \mathrm{mg}$ /day to $8.9 \pm$ $6.6 \mu \mathrm{g} / \mathrm{mL}$ for $1200 \mathrm{mg} / \mathrm{day}^{28}$
Pharmacokinetic interactions have been observed between eslicarbazepine and other commonly used antiepileptic drugs, similar to interactions seen with oxcarbazepine (Table 1). ${ }^{29}$ Steady state levels of eslicarbazepine were associated with a significant increase in both phenytoin and phenobarbital, possibly due to inhibition of the hepatic enzyme CYP2C19. ${ }^{29}$ Conversely, phenytoin and phenobarbital increased clearance of eslicarbazepine, and a 33\% decrease in eslicarbazepine exposure was noted with concomitant phenytoin therapy. ${ }^{29}$ Small, possibly insignificant increases in clearance of topiramate, lamotrigine and carbamazepine were seen with concomitant use of eslicarbazepine, while a significant increase in eslicarbazepine clearance was noted with concomitant carbamazepine therapy. ${ }^{29}$ Other commonly used medications which may be affected by eslicarbazepine include warfarin and hormonal contraceptives. ${ }^{12}$ Eslicarbazepine may lower levels of ethinylestradiol, possibly interfering with the efficacy of certain hormonal contraceptives, and may increase levels of tolbutamide, a first generation sulfonylurea. ${ }^{12}$ There are no kinetic interactions between eslicarbazepine and digoxin, ${ }^{30}$ or metformin ${ }^{31}$ when studied in healthy subjects taking both medications.

\section{Efficacy studies}

Eslicarbazepine has been shown to be effective in several animal models of epilepsy. In maximal electroshock seizures, eslicarbazepine was equal to carbamazepine, and superior to oxcarbazepine at 2 and 4 hours after the dose. At 8 hours after dose, all three drugs were equivalent. ${ }^{12}$ Eslicarbazepine was equivalent to carbamazepine in prevention of seizures in the amygdala kindling model. ${ }^{12}$ Furthermore, eslicarbazepine has been shown to be effective in the control of seizures induced

Table I Summary of potential pharmacokinetic interactions between eslicarbazepine and other medications ${ }^{29}$

\begin{tabular}{lll}
\hline & $\begin{array}{l}\text { Effect of eslicarbazepine } \\
\text { on other drugs }\end{array}$ & $\begin{array}{l}\text { Effect of other drugs } \\
\text { on eslicarbazepine }\end{array}$ \\
\hline Phenytoin & $\uparrow \uparrow$ & $\downarrow$ \\
Phenobarbital & $\uparrow \uparrow$ & $\downarrow$ ? \\
Topiramate & $\downarrow$ ? & $\leftrightarrow$ \\
Lamotrigine & $\downarrow$ ? & $\leftrightarrow$ \\
Carbamazepine & $\downarrow$ ? & $\downarrow$ \\
Valproate & $\leftrightarrow$ & $\leftrightarrow$ \\
Levetiracetam & $\leftrightarrow$ & $\leftrightarrow$ \\
Warfarin & $\downarrow$ ? & $\leftrightarrow$ \\
Ethinylestradiol & $\downarrow$ & $?$ \\
Tolbutamide & $\uparrow$ & $?$ \\
\hline
\end{tabular}


by proconvulsant agents such as metrazole, bicuculline, 4-amino-pyridine, ${ }^{12}$ latruncullin $\mathrm{A},{ }^{32}$ and picrotoxin. ${ }^{33}$

In humans, the clinical trials have focused on partial-onset seizures. In the first published study, 143 patients with $\geq 4$ partial onset seizures/month, were recruited in a 12-week, multicenter, randomized, double-blind, placebo-controlled comparison of eslicarbazepine once daily $(n=50)$, twice daily $(n=46)$, or placebo $(n=47)$ added to their existing anticonvulsant regimen. ${ }^{34}$ The dosage of eslicarbazepine was increased every 4 weeks from $400 \mathrm{mg} /$ day to $800 \mathrm{mg} /$ day, to $1200 \mathrm{mg} /$ day. One hundred thirteen $(78.5 \%)$ patients completed the study. Mean age was approximately 39 , and mean body mass index (BMI) was approximately 25 . Nearly half $(40.6 \%)$ were male. ${ }^{34}$

Partial seizures with secondary generalization were the most common (77.6\%), followed by complex partial seizures $(74.8 \%)$, and simple partial seizures $(32.9 \%)$. Concomitant anticonvulsants consisted of: valproic acid $(65.0 \%)$, topiramate $(30.8 \%)$, lamotrigine $(30.1 \%)$, clonazepam $(15.4 \%)$, and phenytoin (7.7\%). Patients receiving oxcarbazepine were excluded from the study. Phenytoin was over represented in the placebo group (17\% of placebo treated patients) compared to single daily dose group (2\%) or twice daily dose group (4\%). ${ }^{34}$

Response was defined as a minimum of $50 \%$ reduction in seizure frequency versus baseline. Single daily dosed eslicarbazepine was more effective than placebo (54\% vs $28 \%$, respectively; $90 \% \mathrm{CI}=-\infty,-14, P=0.008)$. Twice daily dosing was not significantly better than placebo (41\% vs $28 \%$, respectively; $90 \% \mathrm{CI}=-\infty,-1, P=0.12$ ). The greatest reduction in seizure number occurred in subjects receiving a single dose of $1200 \mathrm{mg} /$ day with a $60 \%$ reduction compared to a $30 \%$ reduction in those receiving placebo $(P=0.002) .{ }^{34}$ Elimination of seizures occurred in $24 \%$ of those receiving eslicarbazepine compared to only $9 \%$ of those receiving placebo $(P<0.05){ }^{34}$

In a similar, larger study, 402 patients were recruited in a 12-week, randomized, double-blind, placebo-controlled study of single daily dose of $400 \mathrm{mg}(\mathrm{n}=100), 800 \mathrm{mg}(\mathrm{n}=$ 98), $1200 \mathrm{mg}(\mathrm{n}=102)$, or placebo $(\mathrm{n}=102) .{ }^{28}$ Of the 402 patients, $330(82 \%)$ completed the study. The mean age was 39 years (range $18-76$ years). Half (51\%) were women, and all were Caucasian. The mean BMI was 24.5. Most patients were receiving 2 concomitant anticonvulsants $(n=257$, $63.9 \%$ ), only 2 were receiving 3 anticonvulsants, and the remainder $(n=143,35.6 \%)$ were taking only one concomitant anticonvulsant. The most common concomitant anticonvulsant was carbamazepine (58.5\%), followed by lamotrigine (26.1\%), valproic acid (25.6\%), topiramate $(13.7 \%)$, levetiracetam $(8.5 \%)$, phenobarbital $(8.2 \%)$, clonazepam $(7.5 \%)$, and gabapentin (5.5\%) (oxcarbazepine excluded). ${ }^{28}$
The most common seizure type was complex partial seizures (69.7\%). Nearly half of the patients had secondarily generalized seizures $(46.3 \%)$ and simple partial seizures $(44.0 \%)$. A small fraction had unclassified seizures $(4.5 \%){ }^{28}$ Duration of epilepsy was approximately 21 years, and the mean baseline seizure frequency was 2.9 seizures/week.

There was a dose related increase in the fraction of patients who responded ( $\leq 50 \%$ reduction in seizure frequency). Twenty percent of placebo-treated patients responded, compared to $23 \%$ of those receiving eslicarbazepine $400 \mathrm{mg} /$ day (ns), $34 \%$ of $800 \mathrm{mg} /$ day $(P<0.05)$, and $43 \%$ of $1200 \mathrm{mg} /$ day $(P<0.001) .{ }^{28}$ The likelihood of being free of seizures was only slightly higher with eslicarbazepine compared to placebo ( $8 \%$ of those receiving $1200 \mathrm{mg} /$ day, $P<0.05,4 \%$ of those receiving $800 \mathrm{mg} /$ day, $\mathrm{ns}, 2 \%$ of those receiving $400 \mathrm{mg} /$ day, ns, and $2 \%$ of those receiving placebo). ${ }^{28}$ The statistical methodology used in this study of the last observation carried forward generally underestimates the utility of the drug.

There is only one published study in a paediatric population. ${ }^{35}$ Eleven young children (ages $2-7$ years, mean age $4.1 \pm \mathrm{SD} 1.4$ years), 8 older children (7-11 years, mean age $9.1 \pm 1.6$ years), and 10 adolescents (12-17 years, mean age $14.5 \pm 1.6$ years) were given single daily doses of eslicarbazepine in an open fashion. Forty-one percent were male. The majority were on 3 concomitant anticonvulsants (55.2\%), nearly one-third were on 2 anticonvulsants (27.6\%), and the rest were on 1 anticonvulsant (17.2\%). The most common concomitant agents were lamotrigine $(65.5 \%)$ and valproic acid $(65.5 \%)$, followed by topiramate $(48.3 \%)$, clonazepam (27.6\%), phenobarbital (13.8\%), and gabapentin (10.3\%). ${ }^{35}$

Eslicarbazepine doses were initiated at $5 \mathrm{mg} / \mathrm{kg} /$ day for 4 weeks, and increased to $15 \mathrm{mg} / \mathrm{kg} /$ day for an additional 4 weeks, and then to $30 \mathrm{mg} / \mathrm{kg} / \mathrm{day}$ (or $1800 \mathrm{mg} / \mathrm{day}$, whichever was less) for the last 4 weeks. ${ }^{35}$ Young children and adolescents experienced a dose related reduction in seizure frequency. In young children there was a $28.2 \%, 24.8 \%$, and $40.6 \%$ reduction with doses of 5,15 , and $30 \mathrm{mg} / \mathrm{kg} / \mathrm{day}$, respectively. In adolescents there was a $17.1 \%, 31.7 \%$, and $43.1 \%$ reduction in seizure frequency with ascending doses. This pattern was not observed in older children $(11.7 \%, 5.0 \%$, and $12.2 \%$ reduction in seizure frequency at doses of 5,15 , and $30 \mathrm{mg} / \mathrm{kg} /$ day). ${ }^{35}$

\section{Safety and tolerability}

Eslicarbazepine does not form the 10-11 epoxycarbamazepine metabolite that is formed when carbamazepine is prescribed. Consequently, it does not carry the same adverse consequences of that drug. However, when given 
Table 2 Summary of percentage of adverse events across two placebo-controlled trials ${ }^{27,33}$

\begin{tabular}{llllll}
\hline $\begin{array}{l}\text { Adverse } \\
\text { event (AE) }\end{array}$ & Placebo & $\begin{array}{l}\text { Eslicarbazepine } \\
\mathbf{4 0 0} \mathbf{~} \text { g/day }\end{array}$ & $\begin{array}{l}\text { Eslicarbazepine } \\
\mathbf{8 0 0} \mathbf{~ m} \text { /day }\end{array}$ & $\begin{array}{l}\text { Eslicarbazepine } \\
\text { I 200 } \mathbf{~ m g / d a y}\end{array}$ & $\begin{array}{l}\text { Eslicarbazepine } \\
\text { variable dose }\end{array}$ \\
\hline Any AE & 31.4 & 44.0 & 50.0 & 60.8 & 39.6 \\
Dizziness & 2.0 & 4.0 & 14.3 & 13.7 & 4.1 \\
Headache & 5.9 & 5.0 & 9.2 & 10.8 & 5.2 \\
Diplopia & 0 & 2.0 & 7.1 & 10.8 & 0 \\
Somnolence & 2.0 & 6.0 & 9.2 & 9.8 & 4.1 \\
Vertigo & 1.0 & 2.0 & 2.0 & 5.9 & 0 \\
AE leading to & 3.9 & 4.0 & 8.2 & 19.6 & 7.3 \\
discontinuation & & & & & \\
\hline
\end{tabular}

as an add-on drug to other anticonvulsants in a placebocontrolled fashion, eslicarbazepine is associated with adverse events (AEs) in $41 \%$ to $61 \%$ of subjects (Table 2 ). In one study of 143 patients, the overall rate of AEs was not significantly different than placebo (ie, concomitant anticonvulsant) (Table 2). ${ }^{34}$ In a study of 402 patients, AEs for the highest dose were twice as common as seen in placebo (ie, concomitant anticonvulsant) (Table 2). ${ }^{28}$ The likelihood of adverse consequence is dose related with a higher rate of discontinuation for $\mathrm{AE}$ at higher doses (Table 2). The adverse effect profile is similar to that seen with both carbamazepine and oxcarbazepine and likely reflects a common mechanism of action. In all of the studies, there was only one death, a placebo-treated patient (ie, receiving concomitant anticonvulsants) who died from hypothermia. Electrocardiogram changes, specifically a mild prolongation of the PR interval, have been observed in patients taking eslicarbazepine. ${ }^{29}$ In recommending approval of eslicarbazepine in Europe, the European Medicines Agency advised a contraindication to the administration of eslicarbazepine to patients with 2nd or 3rd degree heart block and caution with co-administration of any drugs known to increase the PR interval. ${ }^{29}$

There were no significant changes in blood cell parameters or serum sodium levels. Among the two placebo-controlled studies, there were 4 cases of abnormal laboratory findings. One case of hyponatremia (123 mmol/L) occurred in a patient receiving eslicarbazepine $800 \mathrm{mg}$ once daily with concomitant carbamazepine and a baseline sodium of $133 \mathrm{mmol} / \mathrm{L} .{ }^{28}$ Additionally there was one case each of mild electrolyte imbalance, mild anemia, and a moderate elevation of creatine kinase. ${ }^{34}$

The overall plasma levels of eslicarbazepine varied in a linear fashion with dose over the therapeutic dose range (Table 3). ${ }^{28}$ None of the studies reported side effects as a function of concomitant anticonvulsant medication. This is an important point since AEDs with a similar mechanism (sodium channel blockade) might have an additive effect on observed AEs.

Among 29 children receiving eslicarbazepine in addition to other anticonvulsants, 21 subjects reported 54 AEs. ${ }^{35}$ The frequency of reported AEs varied with dose so that 11 occurred with low dose of $5 \mathrm{mg} / \mathrm{kg} /$ day, 15 occurred with the moderate dose of $15 \mathrm{mg} / \mathrm{kg} / \mathrm{day}$, and 28 were reported with the high dose of $30 \mathrm{mg} / \mathrm{kg} /$ day. ${ }^{35}$ All AEs at lower doses were mild. At $30 \mathrm{mg} / \mathrm{kg} /$ day there were 9 moderate AEs and 2 severe AEs. Moderate AEs included somnolence $(n=3)$, vomiting $(n=2)$, diplopia $(n=2)$, dizziness $(n=1)$, and disequilibrium $(n=1)$. The severe cases were both related to worsening seizure disorder. ${ }^{35}$

\section{Conclusions}

Eslicarbazepine acetate is a prodrug which is rapidly and almost completely metabolized to the active metabolite (S) licarbazepine. It is a member of the dibenzazepine family, which includes carbamazepine and oxcarbazepine. In animal studies, it has similar potency to the active metabolite of carbamazepine with fewer adverse effects and interactions, including cytochrome oxidases. Its preferential binding to VGSC in the inactivated state should result in selective inhibition of the most rapidly firing sodium channels, thereby limiting the excessive neuronal activity of seizures. It does not form the epoxide intermediate which is thought to be responsible for many of the adverse effects of carbamazepine. Its estimated half-life of 20-24 hours makes once-daily dosing a possibility.

Table 3 Eslicarbazepine levels as a function of $\operatorname{dose}^{27}$

\begin{tabular}{llll}
\hline & $\mathbf{4 0 0} \mathbf{~ m g} /$ day & $\mathbf{8 0 0} \mathbf{~ m g} /$ day & I $200 \mathbf{~ m g / d a y}$ \\
\hline $\begin{array}{l}\text { Eslicarbazepine } \\
\text { level }(\mu \mathrm{g} / \mathrm{mL})\end{array}$ & $2.4 \pm 2.2$ & $4.7 \pm 4.0$ & $8.9 \pm 6.6$ \\
\hline
\end{tabular}


Dosage adjustments will be necessary in patients with moderate to severe renal impairment. Eslicarbazepine may affect levels of other medications including phenytoin, warfarin and estrogen-containing oral contraceptives, and certain enzyme-inducing AEDs may lower levels of eslicarbazepine. A contraindication to eslicarbazepine has been recommended in patients with 2 nd or 3rd degree heart block, otherwise eslicarbazepine appears to be very safe. Efficacy has been demonstrated in at least three clinical trials of eslicarbazepine as adjunctive therapy for seizures, with a significant reduction in seizures as compared to placebo. Eslicarbazepine was welltolerated in these trials, with the most common side effects being dizziness, nausea, vomiting, somnolence and diplopia. Hyponatremia, an idiosyncratic reaction occasionally seen with oxcarbazepine, was only reported in one study patient who was on both eslicarbazepine and carbamazepine.

The published studies investigated a relatively small number of patients with few comorbid medical problems. The effectiveness of eslicarbazepine in a typical clinical population still needs to be investigated. Initial use of eslicarbazepine will likely be as adjunctive therapy in adults with complex partial seizures who may be on a variety of other medications, including those affected by the CYP3A4 (including certain calcium channel blockers and zonisamide) and the CYP2C19 enzyme systems. Additionally, an examination of the side effect profile may be warranted on patients on other psychotropics (eg, antidepressants or antipsychotic medications) or cardiovascular agents that may accentuate the AE profile of eslicarbazepine. Finally, a study of endocrinologic effects of ESL may be warranted as changes in thyroid function ${ }^{36,37}$ and vitamin D levels ${ }^{38,39}$ have been observed in adults and children on oxcarbazepine and decreased bone mineral density has been observed in pediatric patients receiving oxcarbazepine. ${ }^{39}$

In the past few years, as AEDs with less toxicity and fewer interactions have become available, emphasis on monotherapy in treatment resistant epilepsy has begun to give way to a sort of 'rational polytherapy' as means of controlling seizures, ${ }^{40,41}$ by using smaller doses of a combination of AEDs with different mechanisms of action to attain the goal of complete seizure control with fewer side-effects. As a group, the sodium channel blockers are among the most frequently prescribed medications in the management of epilepsy. Although a direct comparison has not been performed, eslicarbazepine may be better tolerated and more effective in controlling partial onset seizures than oxcarbazepine. The added benefit of once-daily dosing, which is not possible with carbamazepine or oxcarbazepine, may improve patient compliance with therapy and thereby further improve seizure control. Eslicarbazepine appears to be a safe and effective addition to the armamentarium of physicians managing patients with epilepsy.

\section{Disclosures}

The authors declare no conflicts of interest.

\section{References}

1. Hauser WA, Annegers JF, Kurland LT. Prevalence of epilepsy in Rochester, Minnesota: 1940-1980. Epilepsia. 1991;32(4): 429-445.

2. Hauser WA, Annegers JF, Kurland LT. Incidence of epilepsy and unprovoked seizures in Rochester, Minnesota: 1935-1984. Epilepsia. 1993;34(3):453-468.

3. Forsgren L, Beghi E, Oun A, Sillanpaa M. The epidemiology of epilepsy in Europe - a systematic review. Eur J Neurology. 2005;12: 245-253.

4. Birbeck GL Hays RD, Cui XP, Vickrey BG. Seizure Reduction and Quality of Life Improvements in People with Epilepsy. Epilepsia. 2002;43(5):535-538.

5. Wirrell EC. Epilepsy-related Injuries. Epilepsia. 2006;47(Suppl 1): 79-86.

6. Josty IC, Narayanan V, Dickson WA. Burns in patients with epilepsy: changes in epidemiology and implications for burn treatment and prevention. Epilepsia. 2000;41(4):453-456.

7. Hamer HM, Spottke A, Aletsee C, et al. Direct and indirect costs of refractory epilepsy in a tertiary epilepsy center in Germany. Epilepsia. 2006;47(12):2165-2172.

8. Krauss GL, Ampaw L, Krumholz A. Individual state driving restrictions for people with epilepsy in the US. Neurology. 2001;57(10): $1780-1785$.

9. Kwan P, Brodie MJ. Early identification of refractory epilepsy. N Engl J Med. 2000;342(5):314-319.

10. Faught E. Monotherapy in adults and elderly persons. Neurology. 2007;69(Suppl 3):S3-S9.

11. Almeida L, Falcão A, Maia J, Mazur D, Gellert M, Soares-da-Silva P. Single-dose and steady-state pharmacokinetics of eslicarbazepine acetate (BIA 2-093) in healthy elderly and young subjects. J Clin Pharmacol. 2005;45:1062-1066.

12. Almeida L, Soares-da-Silva P. Eslicarbazepine acetate (BIA 2-093). Neurotherapeutics. 2007;4:88-96.

13. Volosov A, Xiaodong S, Perucca E, Yagen B, Sintov A, Bialer M. Enantioselective pharmacokinetics of 10-hydroxycarbazepine after oral administration of oxcarbazepine to health Chinese subjects. Clin Pharmacol Ther. 1999;66(6):547-553.

14. Benes J, Parada A, Figueiredo AA, et al. Anticonvulsant and sodium channel-blocking properties of novel 10,11-dihydro- $5 \mathrm{H}$ dibenz $[b, f]$ azepine-5-carboxamide derivatives. J Med Chem. 1999; 42(14):2582-2587.

15. Yamaoka K, Vogel SM, Seyama I. $\mathrm{Na}^{+}$channel pharmacology and molecular mechanisms of gating. Curr Pharm Des. 2006; 12(4):429-442.

16. Parada A, Soares-da-Silva P. The novel anticonvulsant BIA 2-093 inhibits transmitter release during opening of voltage-gated sodium channels: a comparison with carbamazepine and oxcarbazepine. Neurochem Int. 2002;40:435-440.

17. Bonifácio MJ, Sheridan RD, Parada A, Cunha RA, Patmore L, Soares-da-Silva P. Interaction of the novel anticonvulsant, BIA 2-093, with voltage-gated sodium channels: comparison with carbamazepine. Epilepsia. 2001;42(5):600-608.

18. El-Mallakh RS, Huff MO. Mood stabilizers and ion regulation. Harv Rev Psychiatry. 2001;9:23-32. 
19. Araújo IM, Ambrósio AF, Leal EC, et al. Neurotoxicity induced by antiepileptic drugs in cultured hippocampal neurons: a comparative study between carbamazepine, oxcarbazepine, and two new putative antiepileptic drugs, BIA 2-024 and BIA 2-093. Epilepsia. 2004; 45(12):1498-1505.

20. Fontes-Ribeiro C, Nunes T, Falcäo A, et al. Eslicarbazepine acetate (BIA 2-093): Relative bioavailability and bioequivalence of $50 \mathrm{mg} / \mathrm{mL}$ oral suspension and $200 \mathrm{mg}$ and $800 \mathrm{mg}$ tablet formulations. Drugs R D. 2005;6(5):253-260.

21. Maia J, Vas-da-Silva M, Almeida L, et al. Effect of food on the pharmacokinetic profile of eslicarbazepine acetate (BIA 2-093). Drugs R D. 2005;6(4):201-206.

22. Fontes-Ribeiro C, Macedo T, Nunes T, et al. Dosage form proportionality and food effect of the final tablet formulation of eslicarbazepine acetate: randomized, open-label, crossover, single-centre study in healthy volunteers. Drugs R D. 2008;9(6):447-454.

23. Falcão A, Maia J, Almeida L, Mazur D, Gellert M, Soares-da-Silva P Effect of gender on the pharmacokinetics of eslicarbazepine acetate (BIA 2-093), a new voltage-gated sodium channel blocker. Biopharm Drug Dispos. 2007;28(5):249-256.

24. Almeida L, Potgieter JL, Maia J, Potgieter MA, Mota F, Soares-da-Silva P. Pharmacokinetics of eslicarbazepine acetate in patients with moderate hepatic impairment. Eur J Clin Pharmacol. 2008;64(3):267-273.

25. Almeida L, Soares-da-Silva P. Safety, tolerability and pharmacolokinetic profile of BIA 2-093, a novel putative antiepileptic, in a rising multiple-dose study in young healthy humans. J Clin Pharmacol. 2004;44:906-918.

26. Maia J, Almeida L, Falcão A, et al. Effect of renal impairment on the pharmacokinetics of eslicarbazepine acetate. Int J Clin Pharmacol Ther. 2008;46(3):119-120.

27. Alves G, Figueiredo I, Castel-Branco M, et al. Biomed Chromatogr. 2007;21(11):1127-1134

28. Elger C, Halász P, Maia J, Almeida L, Soares-da-Silva P, BIA-2093-301 Investigators Study Group. Efficacy and safety of eslicarbazepine acetate as adjunctive treatment in adults with refractory partial-onset seizures: A randomized, double blind, placebo-controlled, parallelgroup phase III study. Epilepsia. 2009;50(3):454-463.

29. European Medicines Agency. CHMP Assessment Report for Exalief: International Nonproprietary Name: eslicarbazepine acetate. London, 19 February 2009 http://www.emea.europa.eu/humandocs/PDFs/EPAR/ exalief/H-987-en6.pdf, accessed 13 October 2009.
30. Vas da Silva M, Costa R, Soares E, et al. Effect of eslicarbazepine acetate on the pharmacokinetics of digoxin in healthy subjects. Fundam Clin Pharmacol. 2009. Epub ahead of print.

31. Rocha JF, Caz-da-Silva M, Almeida L, et al. Effect of eslicarbazepine acetate on the pharmacokinetics of metformin in healthy subjects. Int J Clin Pharmacol Ther. 2009;47(4):255-261.

32. Sierra-Paredes G, Oreiro-García T, Vázquez-Illanes MD, Sierra-Marcuňo G. Effect of eslicarbazepine acetate (BIA 2-093) on latrunculin A-induced seizures and extracellular amino acid concentrations in the rat hippocampus. Epilepsy Res. 2007;77(1):36-42.

33. Sierra-Paredes G, Núňez-Rodriguez A, Vázquez-López A, Oreiro-García T, Sierra-Marcuňo G. Anticonvulsant effect of eslicarbazepine acetate (BIA 2-093) on seizures induced by microperfusion of picrotoxin in the hippocampus of freely moving rats. Epilepsy Res. 2006;72(2-3):140-146.

34. Elger C, Bialer M, Cramer JA, Maia J, Almeida L, Soares-da-Silva P. Eslicarbazepine acetate: A double-blind, add-on, placebo-controlled, exploratory trial in adult patients with partial-onset seizures. Epilepsia. 2007;48(3):497-504.

35. Almeida L, Minciu I, Nunes T, et al. Pharmacokinetics, efficacy, and tolerability of eslicarbazepine acetate in children and adolescents with epilepsy. J Clin Pharmacol. 2008;48(8):966-977.

36. Isojarvi JI, Turkka J, Pakarinen AJ, Kotila M, Rattya J, Myllyla VV. Thyroid function in men taking carbamazepine, oxcarbazepine, or valproate for epilepsy. Epilepsia. 2001;42(7):930-934.

37. Hirfanoglu T, Serdaroglu A, Camurdan O, et al. Thyroid function and volume in epileptic children using carbamazepine, oxcarbazepine and valproate. Pediatrics International. 2007;49(6):822-826.

38. Mintzer S, Boppana P, Toguri J, DeSantis A. Vitamin D levels and bone turnover in epilepsy patients taking carbamazepine or oxcarbazepine. Epilepsia. 2006;47(3):510-515.

39. Cansu A, Yesilkaya E, Serdarog ${ }^{\complement}$ lu A, et al. Evaluation of bone turnover in epileptic children using oxcarbazepine. Pediatr Neurol. 2008;39:266-271.

40. Deckers C, Czuczwar S, Hekster Y, et al. Selection of antiepileptic drug polytherapy based on mechanisms of action: the evidence reviewed. Epilepsia. 2000;41(11):1364-1374.

41. Leppik IE. Monotherapy and polypharmacy. Neurology. 2000; 55(11 Suppl 3):S25-S29.
Therapeutics and Clinical Risk Management

\section{Publish your work in this journal}

Therapeutics and Clinical Risk Management is an international, peerreviewed journal of clinical therapeutics and risk management, focusing on concise rapid reporting of clinical studies in all therapeutic areas, outcomes, safety, and programs for the effective, safe, and sustained use of medicines. This journal is indexed on PubMed Central, CAS,

\section{Dovepress}

EMBase, Scopus and the Elsevier Bibliographic databases. The manuscript management system is completely online and includes a very quick and fair peer-review system, which is all easy to use. Visit http://www.dovepress.com/testimonials.php to read real quotes from published authors. 